\title{
Bidirectional orbital approach enhances orbital abscess drainage*
}

\author{
Ephraim Eviatar, Alex Kessler, Koby Pitaro \\ Department of Otorhinolaryngology Head and Neck Surgery, Assaf Harofeh Medical Center, Zerifin 70300, \\ Israel, affiliated to the Sackler Faculty of Medicine, Tel Aviv University, Ramat Aviv, Israel
}

\begin{abstract}
SUMMARY
Objectives: Transnasal endoscopic management of subperiosteal orbital abscess (SPOA) secondary to acute rhinosinusitis has become very popular over the past two decades. We describe our transnasal endoscopic approach for orbital complications secondary to acute rhinosinusitis (SPOA and orbital abscess) and the efficacy criteria that can be used to ensure complete drainage.

Methods: The charts of all patients who underwent surgery at Assaf Harofeh Medical Center between January 1993 and June 2007 were reviewed. Age, sex, clinical findings, antibiotic treatment, surgical procedure and outcome were recorded.

Results: Nineteen patients with SPOA and three with orbital abscess underwent surgery during which wide exposure of the periorbita was performed. If the abscess was not identified, bidirectional orbital manipulation, while simultaneously keeping a seeker in the abscess space under endoscopic view, enhanced its identification and successful drainage. An immediate reduction in palpable orbital pressure was used as an efficacy criterion for adequate drainage.

Conclusion: The transnasal endoscopic approach for SPOA and orbital abscess can be enhanced by wide exposure of the periorbita and bidirectional orbital manipulation. Keeping track of orbital pressure during surgery by palpating the eye can be used as an efficacy criterion for assessing adequate drainage.
\end{abstract}

Key words: rhinosinusitis, orbital complications, functional endoscopic sinus surgery, treatment, subperiosteal abscess of the orbit, orbital manipulation, orbital abscess

\section{INTRODUCTION}

Acute rhinosinusitis (ARS) may involve the orbit. If not treated promptly and appropriately, orbital involvement may result in acute and permanent blindness or even death ${ }^{(1-3)}$. Several classification systems exist for orbital involvement secondary to ARS. The most popular one is Chandler's classification, which divides orbital complications (OC) into five stages ${ }^{(4)}$. Another classification approach divides OC into those that are preseptal, (Chandler's Stage 1) and those that involve the postseptal orbital content (Chandler's Stage 2 to 4$)^{(5)}$. It is accepted that preseptal cellulitis is the most common orbital complication secondary to ARS and that it can be managed successfully with antibiotics and close follow-up even without hospitalization ${ }^{(2,6,7)}$. In cases of visual compromise, worsening or no response to conservative medical treatment, surgical intervention is needed ${ }^{(1,6)}$. Surgical approaches include the external, transnasal endoscopic (TNE), and combined approach. Since the 1990's, the TNE approach has become widely accepted for the treatment of subperiosteal orbital abscess (SPOA) ${ }^{(6-8)}$, while orbital abscess (OA) is still drained mainly by the external or combined approach. The two main surgical problems that the surgeon may encounter with SPOA and OA are precise localization of the abscess and ensuring complete drainage. For this reason, controversy exists concerning the appropriate steps that should be taken during TNE drainage of SPOA. In one study, a complete ethmoidectomy was recommended, leading to wide exposure of the lamina papyracea (LP) with its dehiscence or cracks ${ }^{(8)}$. In another study, a minimal approach was described, comprising opening the medial wall of the bulla ethmoidalis and exposing the most anterior part of the LP $^{(9)}$. Others concluded that the reason for initial endoscopic drainage failure was inadequate removal of the LP ${ }^{(10)}$. When the abscess is located superiorly or laterally, it was recommend that a stent be left in the orbit at the end of the procedure.

The involved eye has increased pressure compared to the normal eye. This pressure is at its highest at the beginning of surgery, declining during successful drainage until almost normal. The pressure equilibrium between the involved eye and the normal one can be used as a physical efficacy criterion throughout the surgical procedure. We describe our endoscopic approach to 
SPOA and orbital abscess (OA) whereby we perform wide exposure of the periorbita and then use bidirectional orbital manipulation through the nostril. In addition, we follow changes in orbital pressure throughout surgery to ensure adequate drainage.

\section{MATERIALS AND METHODS}

\section{Study protocol}

The study protocol was approved by the Institutional Review Board of our institution.

\section{Patients}

Twenty-two patients who underwent surgery for SPOA and OA at Assaf Harofeh Medical Center between January 1993 and June 2007 were included in the study and their charts were retrospectively reviewed. Age, sex, clinical findings, antibiotic treatment, surgical procedure and outcome were recorded. Surgical intervention was indicated whenever there was a high degree of suspicion for postseptal orbital involvement according to findings on initial ophthalmologic evaluation, which included limitation of globe movement, decreased visual acuity, proptosis or chemosis, any visual deterioration under intravenous antibiotic treatment or no response to conservative management, including persistent fever after the first 48 hours.

\section{Surgery}

Enhanced CT scan of the orbits and sinuses with axial and coronal views was obtained in all patients prior to surgery, to confirm orbital involvement. TNE surgery was performed using $0^{\circ}$ and $30^{\circ}$ endoscopes $(2.7 \mathrm{~mm}$ or $4 \mathrm{~mm}$ ) under general anesthesia in all patients. The procedure began with the placement of cotton pads saturated with 1\% lidocaine and 1:100000 adrenalin in the nasal cavity. Thereafter, the involved eye was palpated and its firmness compared to the uninvolved normal side. The external orbital palpation was performed with great caution in order to prevent the oculocardiac reflex that can lead to bradycardia or even asystole. The lateral nasal wall was injected (1\% lidocaine and 1:100000 adrenalin), the middle turbinate was retracted medially if necessary, uncinectomy was performed, a maxillary antrostomy was created and the anterior and posterior ethmoid cells were completely removed, widely exposing the LP. Dehiscence in the LP was then identified by gentle external manual pressure over the involved eye, forcing pus to flow out through the dehiscence, in order to localize the SPOA. When there was no dehiscence, the LP was cracked with a seeker and removed widely to expose the infected space between the LP and the periorbita and enable abscess drainage. Continuous gentle external pressure was exerted over the orbit (Figure 1A) while manipulating a seeker in the abscess space under endoscopic view until pus no longer exuded (Figure 1B) and orbital firmness was reduced to that of the normal eye. This normalization of orbital pressure was our criterion for complete drainage of the abscess. In cases of sphenoid sinus involvement, the sphenoid sinus was opened in a minimal fashion to drain its contents. No stent was left in place at the end of the procedure because the infected space was widely exposed.
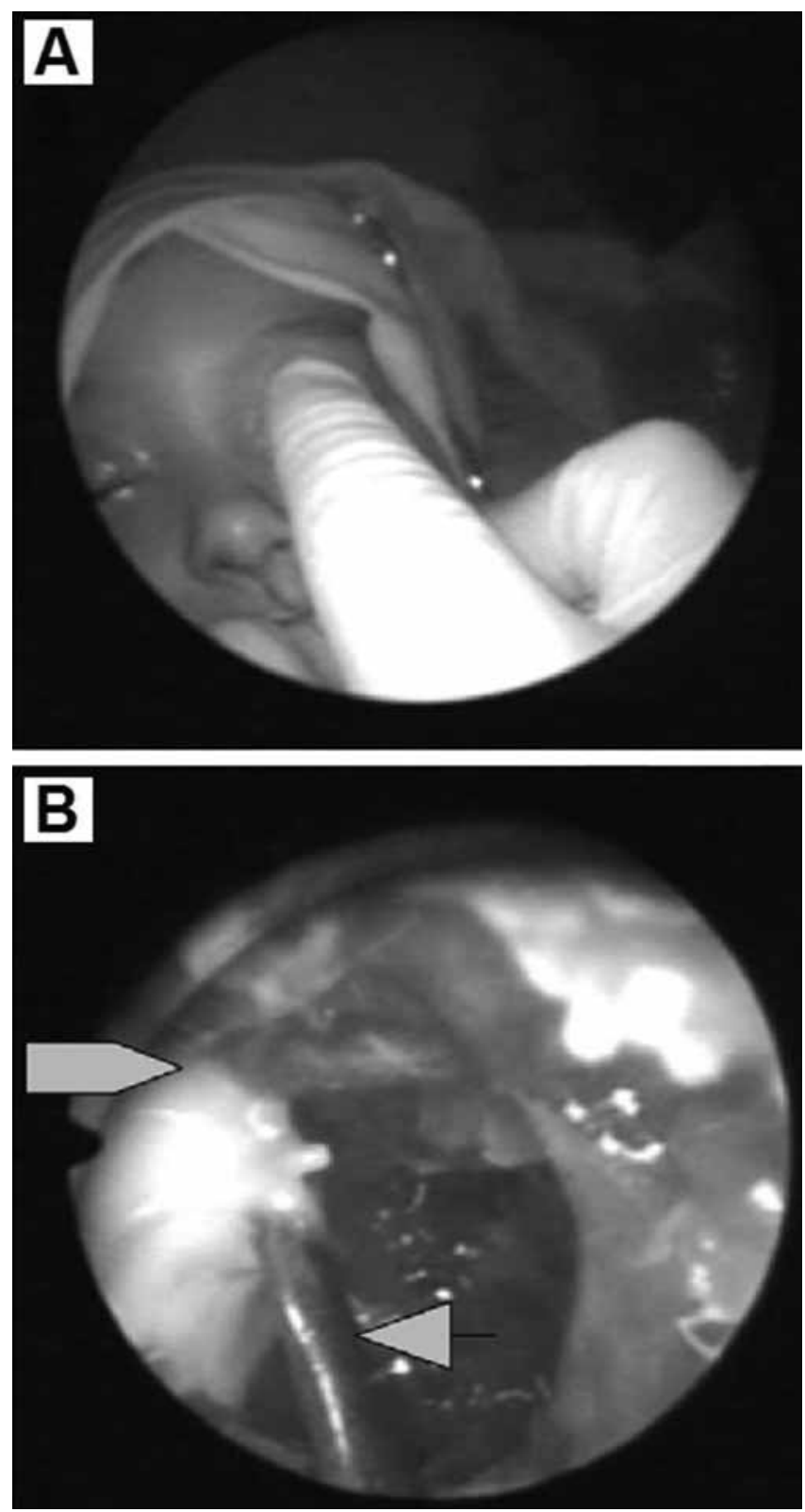

Figure. 1. While manipulating the orbit (A) a seeker is introduced into the abscess space under endoscopic view (B) to enhance complete drainage of the pus. (Lower arrow indicates the seeker in the abscess space, upper arrow indicates pus).

Careful bipolar electrocoagulation was used to achieve hemostasis in order to avoid nasal packing and allow for continuous draining of pus. This was especially important in pediatric patients in whom pack removal can sometimes only be undertaken under general anesthesia. A small nasal pack was left in overnight when bleeding was noticed.

For OA, an incision was made in the periorbita with a sickle knife according to the location of the OA on computerized tomography (CT) scan, and a seeker was introduced into the suspected infected space while synchronously externally manipulating the involved orbit, thereby forcing pus to drain. The manipulation was stopped when the palpated pressure of the involved eye became almost the same as that of the uninvolved eye. 


\section{RESULTS}

Nineteen patients with SPOA and three with OA underwent surgery at our institution. There were 15 males and seven females, aged 2 to 57 years (mean 14 years). Seven patients had oral antibiotic treatment before admission. Once hospitalized, intravenous treatment with cefuroxime or amoxicillin-clavulanic acid was initiated. Neither steroids nor anticongestion preparations were given. In postseptal orbital involvement, limitation of globe movement and proptosis were much more prevalent then in OA (chemosis was only found in OA). The ethmoid was the most common sinus involved on CT scan (19 cases), followed by the maxillary (11 cases), the frontal (three cases), and the sphenoid (two cases). One case of OA that was diagnosed before surgery as SPOA according to CT scan needed revision surgery. The first operation did not yield pus following opening of the subperiosteal space. Revision surgery was performed 12 hours later, during which the OA was successfully drained by incising the periorbita. Neither of the patients with OA needed revision surgery. All patients were operated on via the TNE approach. There was no visual loss or other complications. Cultures from samples taken during surgery revealed Haemophilus influenzae, Staphylococcus aureus, Streptococcus pneumoniae, Moraxella catarrhalis, coagulase-negative Staphylococcus and alphahemolytic Streptococcus. No anaerobes were identified. Following surgery, intravenous antibiotic treatment was continued for at least 48-72 hours with ophthalmologic evaluation twice daily. Patients were discharged on oral antibiotic therapy for a period of 2 weeks. Follow-up at the outpatient clinic was carried out once a week for 1 month. All patients had complete clinical recovery.

\section{DISCUSSION}

Rhinosinusitis continues to be the most common cause of orbital inflammation and infection, especially in children ${ }^{(2)}$. In the preantibiotic era, $17 \%$ of the patients suffering from orbital complications died of meningitis and $20 \%$ became permanently blind in the affected eye ${ }^{(11)}$. Overtime, these rates have declined: the incidence of vision loss reported is $3-11 \%$ and mortality $1-2.5 \%{ }^{(6,12)}$. Currently, the endoscopic approach is the recommended surgical modality of choice because this technique does not require an external incision, produces less postoperative edema, clears the infected sinuses in the direction of the abscess, and allows for faster recovery, and thus a shorter period of hospitalization ${ }^{(8,11)}$.

On the other hand, during ARS the pediatric nasal cavity becomes narrower secondary to the acute inflamed mucosa. In such situations, endonasal endoscopic visualization can easily be disturbed by mucosal bleeding. A multidisciplinary approach is necessary in managing orbital complications secondary to ARS and includes evaluation by an ophthalmologist, an otolaryngologist, a neuroradiologist and an infectious diseases specialist. Patients presenting with eyelid edema must be initially evaluated for preseptal cellulitis or postseptal orbital involvement. Further work up is influenced by the ophthalmologic and clinical status. The clinical features of SPOA are edema, erythema, chemosis and proptosis of the eyelid with limitation of ocular motility, and as a consequence of extra ocular muscle paralysis, the globe becomes fixed (ophthalmoplegia) and visual acuity diminishes ${ }^{(12)}$. CT scans should be obtained whenever surgical drainage is planned.

The management of orbital complications secondary to ARS is mainly conservative (intravenous antibiotics and close follow-up) unless there is reduced globe motility, a threat to vision or no response to antibiotics in the first 48 hours ${ }^{(6)}$. It must be stressed that orbital complications should be treated medically parallel to the surgical treatment because recurrence can occur in cases of inappropriate antibiotic treatment. Cefuroxime or amoxicillin/clavulanic acid constitute an appropriate treatment regimen since they cover the most common causative organisms in ARS. They are often used in our institute since previous experience has shown that many of the patients with orbital complications respond well even without surgery.

Surgical options include the external, endoscopic or combined approach while the endonasal endoscopic approach has become very popular in the last two decades. Several authors have discussed their endoscopic management of orbital complications secondary to ARS.

Manning was the first to describe endoscopic management of medial SPOA ${ }^{(8)}$. He described five pediatric patients with medial SPOA that were successfully managed via an endoscopic ethmoidectomy approach. Four cases were drained through a large dehiscence in the LP and in one case a vertical crack was seen, but no frank dehiscence. Pus exuded after a small portion of bone was elevated, but not removed. The latter patient had a longer hospital stay. The authors concluded that in cases such as this, a small portion of the lateral ethmoid bone should be removed.

Froehlich et al. described their "Minimal Endoscopic Approach" to SPOA ${ }^{(9)}$. They operated on 20 children with SPOA complicating acute ethmoiditis. The surgical procedure was limited to opening the medial wall of the bulla ethmoidalis and partially the LP. Efficacy criteria were partially based on the beginning of eyelid opening at the end of the procedure. It is worth mentioning that in seven patients additional surgical procedures were performed, including adenoidectomy, middle meatal antrostomy and antral lavage.

Immediate LP reconstruction was described by Malik et al. ${ }^{(13)}$ whereby an LP flap was made and mobilized into the nasal cavity posteriorly in a "swing door" pattern without being resected. The flap was repositioned once the eye was clinically decompressed. The rationale behind this technique was the prevention of facial skeleton growth retardation following sinus surgery, although they mentioned that the effect of sinus surgery on facial skeletal growth in humans still needs to be established.

However, there is controversy regarding whether or not the minimal approach is sufficient, whether or not a large portion of the LP should be removed and what other efficacy criteria can be used during the procedure. 
Mann et al. reviewed the reasons for recurrent orbital complications of pediatric sinusitis that failed initial surgical treatment ${ }^{(10)}$. All revision cases were performed endoscopically. The most common reasons for revision surgery were inadequate removal of the LP and a superior or more laterally positioned SPOA. When the abscess was located medially, a portion of the LP was removed and the pus was drained. Revision surgery revealed reaccumulation of pus behind parts of the LP not removed during the initial surgery. One of the reasons for inadequate removal of the anterior portion of the LP was the fear of lacrimal sac injury. They recommended removal of this portion of the LP routinely when initializing the endonasal approach.

Contrary to the minimal approach described previously ${ }^{(9)}$, we widely resect the LP, including its most anterior section. In our series, there were no revisions except the one that was found to be SPOA according to the CT scan, but proved to be OA at surgery. All abscesses were located medially and therefore a superior or lateral abscess was not the case. In our institution, the first step when using the endoscopic approach is to widely expose the LP and look for pus leaking from a dehiscence in the LP, assisted by external manipulation of the globe. If no pus exudes, we crack the LP and remove it widely to expose the periorbita, which will often enable easy drainage of the abscess. If there is still no pus, we then use the bidirectional orbital manipulation maneuver. While keeping a seeker in the abscess space under endoscopic view, the globe is gently palpated, forcing the puss to drain out. As described by Froehlich et al. ${ }^{(9)}$, eyelid opening at the end of the procedure probably indicates a decrease in orbital pressure. We use the change in orbital firmness as an efficacy criterion for ensuring adequate drainage of the abscess. By palpating the eye and comparing it to the normal uninvolved eye at the beginning, during and at the end of the procedure, we are able to decide whether the abscess has been adequately drained. The efficacy of our approach depends on two clinical factors. First, palpating the eye while directly visualizing the surgical field and evaluating the amount of pus that exudes until no further pus drains out, and second, a stepwise decrement in orbital pressure until it equals that of the normal uninvolved eye. The main disadvantage of this clinical method is that there is no objective method for measuring the changes in orbital pressure. Although draining an OA endoscopically has been described less because of its rarity, we managed to treat OA with the same endoscopic technique. Placement of a seeker in the abscess space following opening of the periorbita and use of bidirectional orbital manipulation enabled successful drainage of the abscess. It is extremely important to localize the abscess by carefully reviewing the CT scan to ensure that the opening of the periorbita is as close as possible to the abscess space. Finally, as mentioned by Manning ${ }^{(8)}$, this approach should be considered only when the surgeon is familiar and comfortable with endoscopic sinus surgery, particularly with the use of a seeker inside the orbital fat.
In conclusion, in cases of SPOA and OA, we recommend wide exposure of the periorbita by the removal of a large portion of the LP. Manipulating the orbital content in a bidirectional fashion makes it possible to treat SPOA and OA according to their location on the CT scan. Assessing orbital pressure during the procedure makes it possible to assess the efficacy of the drainage in an adequate manner, although developing a more accurate method to assess the orbital pressure would increase the success of this approach. There were no postoperative complications in our series and all patients were discharged in good health. In our opinion, these two steps should be a part of the endoscopic management of SPOA and OA secondary to ARS.

\section{REFERENCES}

1. American Academy of Pediatrics. Subcommittee on Management of Sinusitis and Committee on Quality Improvement. Clinical practice guideline: management of sinusitis. Pediatrics 2001; 108: 798-808.

2. Fakhri S, Pereira K. Endoscopic management of orbital abscess. Otolaryngol Clin N Am 2006; 39: 1037-1047.

3. Beech T, Robinson A, McDermott AL, Sinha A. Paediatric periorbital cellulitis and its management. Rhinology 2007; 45: 47-49.

4. Chandler JR, Langenbrunner DJ, Stevens ER. The pathogenesis of orbital complications in acute sinusitis. Laryngoscope 1970; 80: 14141428.

5. Mortimore S, Wormald PJ. The Groote Schuur hospital classification of the orbital complications of sinusitis. J Laryngol Otol 1997; 111: 719-723.

6. Younis RT, Lazar RH, Bustillo A, Anand VK. Orbital infection as a complication of sinusitis: are diagnostic and treatment trends changing? Ear Nose Throat J 2002; 81: 771-775.

7. Oxford LE, McClay J. Complications of acute sinusitis in children. Otolaryngol Head Neck Surg 2005; 133: 32-37.

8. Manning SC. Endoscopic management of medial subperiosteal orbital abscess. Arch Otolaryngol Head Neck Surg 1993; 119: 789-791.

9. Froehlich P, Pransky SM, Fontaine P, Stearns G, Morgon A. Minimal endoscopic approach to subperiosteal orbital abscess. Arch Otolaryngol Head Neck Surg 1997; 123: 280-282.

10. Mann W, Amedee RG, Maurer J. Orbital complications of pediatric sinusitis: treatment of periorbital abscess. Am J Rhinol 1997; 11: 149-153.

11. Arjmand EM, Lusk RP, Muntz HR. Pediatric sinusitis and subperiosteal orbital abscess formation: diagnosis and treatment. Otolaryngol Head Neck Surg 1993; 109: 886-894.

12. Fokkens W, Lund V, Mullol J. European position paper on rhinosinusitis and nasal polyps. Rhinol Suppl 2007; 20: 1-136.

13. Malik V, Khwaja S, De Carpentier J. Immediate lamina papyracea reconstruction during endoscopic sinus surgery for surgically managed subperiosteal abscess in children. Laryngoscope 2006; 116: 835838 .

\author{
Ephraim Eviatar, MD \\ Head, Department of Otorhinolaryngology - \\ Head and Neck Surgery \\ Assaf Harofeh Medical Center \\ Zerifin 70300 \\ Israel
}

Tel: +972-8-9779417

Fax: +972-8-9779421

E-mail: fredricag@asaf.health.gov.il 